\section{Endoscopic bridge to radiology: a sequential life-saving hemostatic approach}
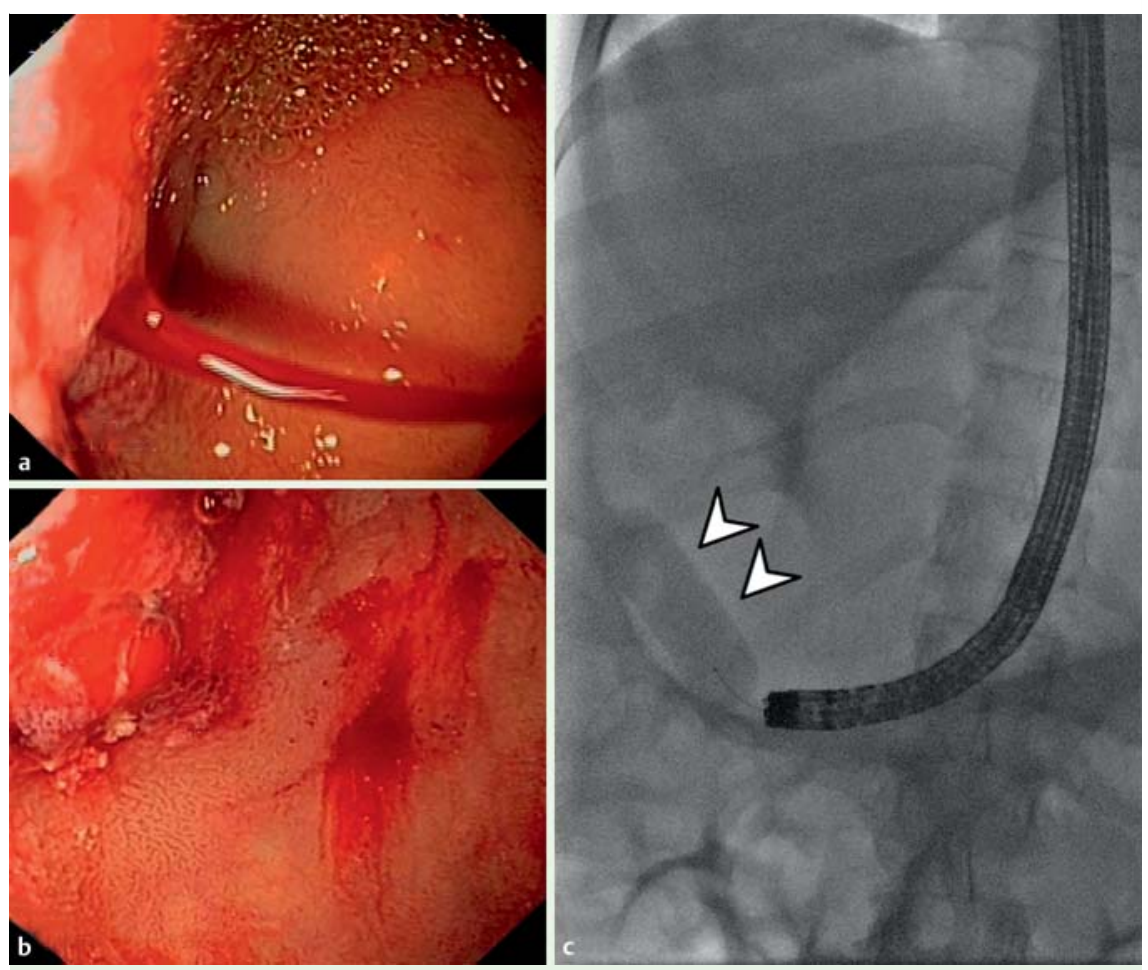

Fig. 1 A 17-year-old girl with massive upper gastrointestinal bleeding underwent an esophagogastroduodenoscopy: a the bleeding varix in the posterior duodenal wall; $\mathbf{b}$ the site of the bleed after glue treatment; c X-ray view of hemostatic balloon (arrowheads) inflated in the duodenal bulb.
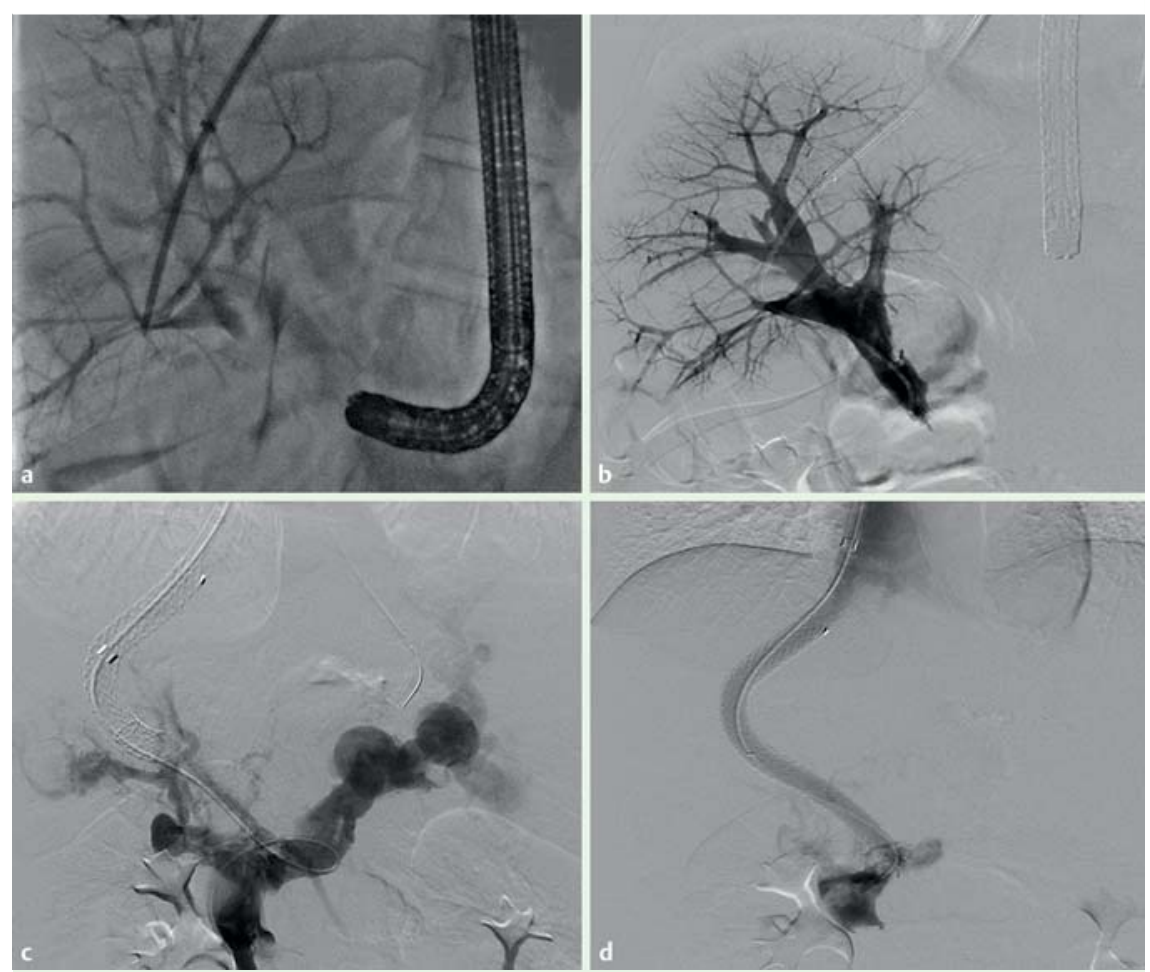

Fig. 2 Emergency transjugular intrahepatic portosystemic shunt was performed. a A Colapinto needle was advanced from the right hepatic vein. $\mathbf{b}$ Contrast dye tracking along the portal vein remnant. c Hydrophilic wire in the splenomesenteric confluence. $\mathbf{d}$ Two stents were deployed.
A 17-year-old girl known to have portal vein cavernoma presented at our institute with massive upper gastrointestinal bleeding, which was causing hypovolemic shock. The girl required multiple blood transfusions and maximal medical support.

Esophagogastroduodenoscopy showed that the source of the bleeding was a varix in the duodenal wall $(\bullet$ Fig. 1 a). The spurting bleed was initially treated with Glubran 2 surgical glue (GEM Srl, Udine, Italy), and although the bleed was reduced, it did not stop ( Fig.1 b). Because of the critical condition of the patient, we decided to inflate a controlled radial expansion balloon (Boston Scientific, Cork, Ireland) to $18 \mathrm{~mm}$ in the duodenal bulb to temporarily stop the bleeding and bridge the patient until radiologic treatment could be provided (๑ Fig. 1 c).

The decision was made to proceed with emergency transjugular intrahepatic portosystemic shunt (TIPS) placement. The site of the bleed was managed endoscopically during the radiological procedure. A Colapinto needle (Ring Transjugular Intrahepatic Access Set; Cook, Bloomington, Indiana, USA) was advanced into the portal vein ( $\bullet$ Fig. $2 \mathrm{a}$ ). Injection of contrast medium showed tracking along the portal vein remnant with stasis due to the absence of communication between the remnant and the splenomesenteric confluence ( Fig.2b). A hydrophilic wire was successfully introduced through the remnant into the splenomesenteric confluence ( Fig.2c). Two stents were deployed in series $(10 \mathrm{~mm}$ polytetrafluoroethylene-covered stents, Viatorr; W.L. Gore, Flagstaff, Arizona, USA) ( $\bullet$ Fig. 2 d). As a result, the portosystemic gradient was reduced from $20 \mathrm{mmHg}$ to $8 \mathrm{mmHg}$. No signs of recurrence were observed in 2 years of endoscopic and radiologic follow-up, during which the patient was in good general condition, and the stents appeared patent on Doppler ultrasound.

The deployment of TIPS in the portal vein remnant may be technically preferable to deployment in the collateral vessels of the cavernoma. This method provides direct communication between the intrahepatic portal radicals and the splenomesenteric confluence, thus creating an effective portosystemic shunt [1-5].

This case highlights how success was achieved by combining the two techniques: the patient was initially stabilized with endoscopic hemostasis, followed by final treatment with radiology. 


\section{Endoscopy_UCTN_Code_TTT_1AR_2AK}

Competing interests: None

\section{Antonino Granata1, *, Roberto Miraglia2, *, Gabriele Curcio ${ }^{1}$, Mario Traina ${ }^{1}$}

${ }^{1}$ Endoscopy Service, Department of Diagnostic and Therapeutic Services, IRCCS - ISMETT (Istituto Mediterraneo per i Trapianti e Terapie ad alta specializzazione), Palermo, Italy

${ }^{2}$ Radiology Service, Department of Diagnostic and Therapeutic Services, IRCCS - ISMETT (Istituto Mediterraneo per i Trapianti e Terapie ad alta specializzazione), Palermo, Italy

\section{References}

1 Fanelli $F$, Angeloni S, Salvatori FM et al. Transjugular intrahepatic portosystemic shunt with expanded-polytetrafluoroethylene-covered stents in non-cirrhotic patients with portal cavernoma. Dig Liver Dis 2011; 43: 78-84

2 Han G, Qi X, He C et al. Transjugular intrahepatic portosystemic shunt for portal vein thrombosis with symptomatic portal hypertension in liver cirrhosis. J Hepatol 2011; 54: $78-88$

3 Senzolo M, Cholongitas E, Tibballs J et al. Relief of biliary obstruction due to portal vein cavernoma using a transjugular intrahepatic portosystemic shunt (TIPS) without the need for long-term stenting. Endoscopy 2006; 38: 760

4 Qi X, Han G, Yin Z et al. Transjugular intrahepatic portosystemic shunt for portal cavernoma with symptomatic portal hypertension in non-cirrhotic patients. Dig Dis Sci 2012; 57: $1072-1082$

5 Di Giorgio A, Agazzi R, Alberti D et al. Feasibility and efficacy of transjugular intrahepatic portosystemic shunt (TIPS) in children. J Pediatr Gastroenterol Nutr 2012; 54: 594 600

\section{Bibliography}

DoI http://dx.doi.org/

10.1055/s-0034-1392211

Endoscopy 2015; 47: E259-E260

(c) Georg Thieme Verlag KG

Stuttgart · New York

ISSN 0013-726X

\section{Corresponding author}

\section{Antonino Granata, MD}

Endoscopy Service, IRCCS - ISMETT

Via Tricomi 5

90127 Palermo, Italy

Fax: +39-091-2192400

agranata@ismett.edu

\footnotetext{
* Both authors contributed equally to this work.
} 